\title{
A rare case of bladder chondroma
}

\author{
Michael D. Carter, MD, PhD; Ricardo A. Rendon, MD, FRCSC; ${ }_{;}^{+}$Jennifer Merrimen, MD, FRCSC*
}

*Anatomical Pathology Division, Queen Elizabeth II Health Science Centre, Halifax, NS; †Department of Urology, Dalhousie University, Halifax, NS

Cite as: Can Urol Assoc J 2015;9(3-4):e136-8. http://dx.doi.org/10.5489/cuaj.2552

Published online March 11, 2015.

\section{Abstract}

Soft tissue chondromas are benign tumours that arise commonly in the hands and feet of adults, but rarely in other locations. Only two prior cases of chondroma of the urinary bladder wall have been reported. We describe a third case and discuss similarities and differences between the three cases reported to date.

\section{Case report}

A 54-year-old woman, with a remote history of tubal ligation but otherwise unremarkable medical history, developed postmenopausal bleeding and right lower quadrant pain. A subsequent pelvic ultrasound was non-contributory with regards to her bleeding; however, an incidental nodule $(1.3 \mathrm{~cm})$ was identified protruding into the bladder from the anterior wall. Referral to Urology was arranged for further investigations and to rule out a possible urothelial carcinoma.

The patient denied any dysuria, hematuria, frequency, urgency, or suprapubic pain. Cytology was negative, showing only degenerated and reactive-appearing urothelial cells. Cystoscopy revealed a firm, uniform, smooth nodular growth on the anterior superior aspect of the bladder. The overlying mucosa was normal and the rest of the bladder was unremarkable. The nodule was entirely removed at the time of cystoscopy using a transurethral resecting loop. The patient tolerated the procedure well and her recovery was uneventful.

The resected specimen was received in the laboratory as numerous fragments of tan-yellow tissue. Microscopic examination showed these to be composed mostly of lobulated, bland, hypocellular, mature hyaline cartilage and two fragments of muscularis propria (Fig. 1). Chondrocytes had small uniform nuclei with no mitotic figures. Immunohistochemical staining of the cells was positive for S100 and vimentin (Fig. 2), and negative for monokeratin (AE1/AE3), Ki-67 and p53. No urothelium was identified in the resected tissue. Resection margins could not be assessed owing to the fragmented nature of the specimen. Collectively, the findings were consistent with a diagnosis of soft tissue chondroma.

Follow-up cystoscopy 5 months after the initial resection failed to reveal any tumours or other abnormalities. The patient's right lower quadrant pain and postmenopausal bleeding resolved in the intervening period.

\section{Discussion}

Soft tissue chondromas are benign tumours that arise commonly in the hands and feet of adults, but rarely in other locations. Only two prior cases of chondroma of the urinary bladder wall have been reported. ${ }^{1,2}$ The most common mesenchymal tumours of the bladder are leiomyomas, leiomyosarcomas, and pleomorphic undifferentiated sarcomas (formerly malignant fibrous histiocytoma). ${ }^{3}$ In recent years there have also been at least 8 reported cases of perivascular epithelioid cell tumour (PEComa) of the bladder. ${ }^{4}$ Chondroid bladder tumours are very rare, with only 2 reported cases of soft tissue chondroma, ${ }^{1,2}$ and 5 of chondrosarcoma. ${ }^{5}$ This diverse group of mesenchymal bladder tumours includes benign and malignant entities, highlighting the importance of their early and accurate diagnosis. Increased awareness of these rare lesions will help towards this end and in the delivery of appropriate care.

Soft tissue chondromas are benign nodules of hyaline cartilage that are not attached to bone and tend to occur in the hands and feet. ${ }^{6}$ Rare occurrences have been reported in various pelvic organs, including the prostate, scrotum, and testis. ${ }^{7}$ They are typically slow-growing, firm, solitary, well-circumscribed, oval-to-round nodules. Microscopic appearance is variable, with about two-thirds consisting of distinct lobules of mature hyaline cartilage. ${ }^{6}$ Local recur- 


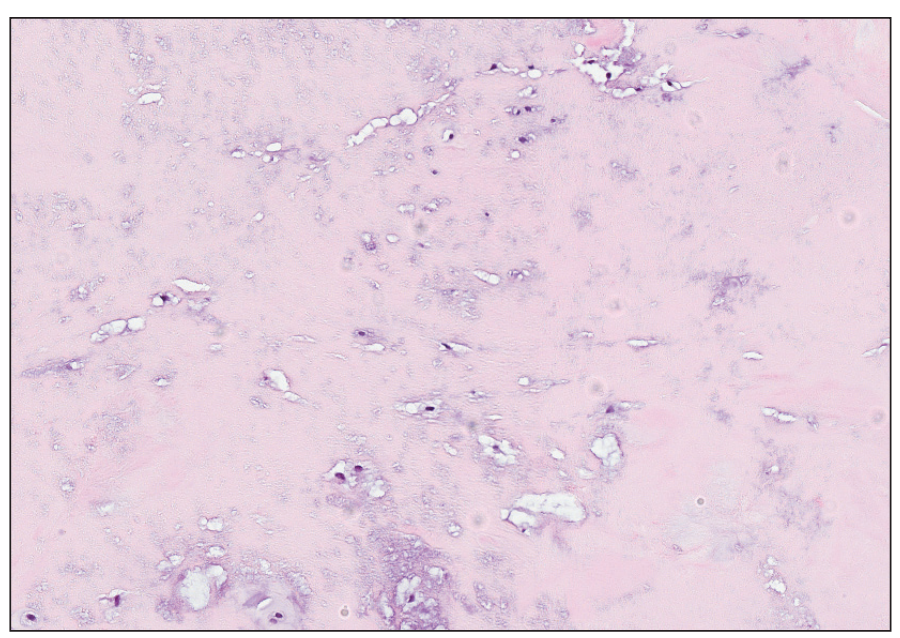

Fig. 1. Fragment of benign hyaline cartilage (hematoxylin and eosin stain, 40× magnification).

rence following excision is seen in about $15 \%$ of cases, but malignant transformation is either exceedingly rare or non-existent. ${ }^{6}$

The patient's right lower quadrant abdominal pain resolved following resection of the bladder chondroma, suggesting it may have been related to the tumour, as reported in an earlier case. ${ }^{2}$ It is highly unlikely, however, that the patient's postmenopausal bleeding, which also resolved in the period following surgery, was related to the tumour.

Computed tomography imaging 4 months after surgery showed a $0.8-\mathrm{cm}$ nodule arising within the bladder wall or extrinsic to the bladder at the site of prior resection. This may represent incomplete resection of the tumour, local recurrence, a small postoperative spindle cell nodule, or simply postoperative scarring. Cystoscopy 1 month later did not reveal any tumours or epithelial abnormalities, as mentioned above. The patient continues to be managed expectantly and is scheduled for a pelvic ultrasound within the next few months.

There are a number of similarities between the two previous cases of bladder chondroma ${ }^{1,2}$ and the one described here. All three cases were in women between the ages of 54 and 63 and all arose on the anterior bladder wall. Upon resection, all had lobulated bland hyaline cartilage that was positive for S100 and vimentin, and negative for AE1/AE3, p53 and Ki-67. The similarities in the ages of the women and the consistent anterior location of their tumours were interesting; however, no conclusions can be drawn from such a small sample size.

The two previous cases of bladder chondroma differ substantially with regards to the clinical settings in which they arose. One of the cases ${ }^{2}$ was associated with pelvic pain but not any urinary symptoms or other bladder pathology, as was seen in the current case. The other bladder chondroma, however, was identified in a woman with a concomitant minimally invasive high-grade urothelial carcinoma of the bladder and symptoms of dysuria, frequency, nocturia, lower abdominal pain, and constipation. ${ }^{1}$ It is not possible to know what role, if any, the coexisting urothelial carcinoma played in the development of the latter patient's chondroma. A connection between these two lesions seems unlikely.

\section{Conclusion}

Soft tissue chondroma of the bladder is a benign subepithelial tumour that has rarely been reported, possibly due to underrecognition and underdiagnosis. Further study of this entity should be pursued once sufficient cases are available; a better understanding of its epidemiology and natural history would help clinicians to identify and treat this rare condition.

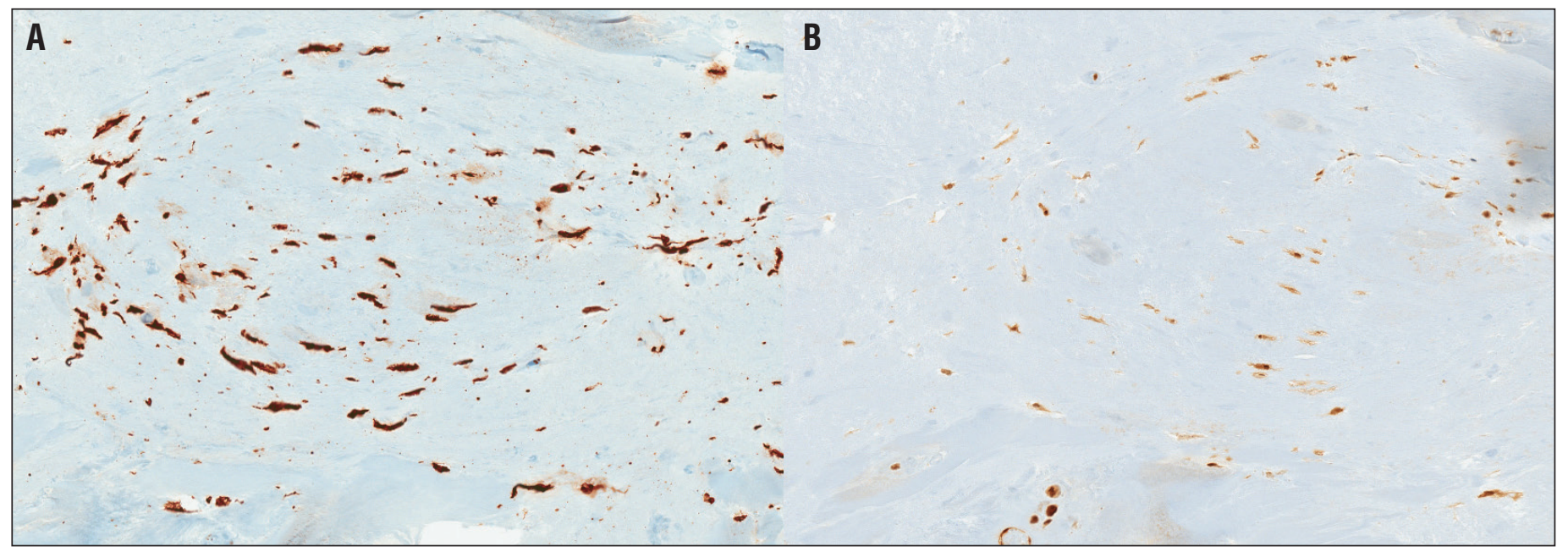

Fig. 2. Positive immunohistochemical staining of chondroma cells with vimentin (A) and $S 100(B)$, both at $40 \times$ magnification. 
Carter et al.

Competing interests: Authors declare no competing financial or personal interests.

This paper has been peer-reviewed.

\section{References}

1. Perrino CM, Pohar KS, Zynger DL. Urinary bladder chondroma. Virchows Arch 2012;460:437-38. hittp:// dx.doi.org/10.1007/s00428-012-1218-y

2. Pauwels CF, Van den Broecke C, Demeyer JM, et al. Chondroma of the bladder. Virchows Arch 1998;432:299-300. hitp://dx.doi.org/10.1007/s004280050169

3. Kunze $E$, Theuring $F$, Krüger $G$. Primary mesenchymal tumors of the urinary bladder: A histological and immunohistochemical study of 30 cases. Pathol Res Pract 1994;190:311-32. http://dx.doi. org/10.1016/S0344-0338(11)80404-8
4. Kyrou D, Staios D, Papatsoris AG. Bladder perivascular epithelioid tumor: A novel rare neoplasm. Urol Int 2012;88:480-2. http://dx.doi.org/10.1159/000332199

5. Ikemoto S, Sugimura K, Yoshida N, et al. Chondrosarcoma of the urinary bladder and establishment of a human chondrosarcoma cell line (OCUU-6). Hum Cell 2004;17:93-6. http://dx.doi. org/10.1111/j.1749-0774.2004.tb00080.x

6. Weiss SW, Goldblum JR (eds). Enzinger and Weiss's Soft Tissue Tumors. 5th edition. Mosby, St. Louis; 2008:1017-23.

7. Reichard C, Robert S, Rubin BP, et al. Scrotal extraosseous chondroma: Case report of an exceedingly unusual presentation and review of the literature. Case Rep Clin Path 2014;1:14-6.

Correspondence: Dr. Michael D. Carter, Anatomical Pathology Division, Queen Elizabeth II Health Science Centre, 6th Floor, Room 638, DJ Mackenzie Building, 5788 University Ave., Halifax, NS B3H 1V8; mcarter5@dal.ca 